\title{
IN SITU MONITORING OF ELECTROEPITAXIAL GROWTH OF THICK AlGaAs LAYERS*
}

\author{
Z.R. ŻYTKIEWICZ \\ Institute of Physics, Polish Academy of Sciences \\ Al. Lotników 32/46, 02-668 Warszawa, Poland
}

\begin{abstract}
In situ growth monitoring technique has been used to analyse growth disturbances during the liquid phase electroepitaxial growth of thick AlGaAs layers. It allowed us to explain the nature of growth instability occurring at the end of the growth and affecting the maximum thickness of AlGaAs layers obtainable by liquid phase electroepitaxy.
\end{abstract}

PACS numbers: $68.55 . \mathrm{Df}, 81.10 . \mathrm{Dn}$

\section{Introduction}

An application of the reflection high-energy electron diffraction (RHEED) technique has made a breakthrough in development of the molecular beam epitaxy (MBE) growth of semiconductor layers. A better understanding of the crystal growth mechanisms and the more precise control of structures parameters was achieved due to an in situ insight into the physical processes occurring on the growing crystal surface. Unfortunately, during the growth from a solution the crystal faces are usually covered by non-transparent melt. Therefore, only post-growth studies of crystal planes morphology or analysis of the shape of striations introduced during the growth procedure can provide one with the information about the behaviour of solid-liquid interface. New possibilities are offered by the liquid phase electroepitaxy (LPEE) - the method in which the dc electric current passing through the solution-substrate interface is a driving force for deposition of the epitaxial layer at constant temperature. LPEE was found to be very effective for the growth of hundreds of micrometers thick and compositionally uniform AlGaAs epilayers $[1,2]$. Moreover, the processes occurring during the growth of such layers can be investigated in detail with the use of in situ monitoring of electroepitaxial growth. This technique was developed by Okamoto et al. [3]. It is based on a simple idea where the resistance of the growing AlGaAs layer contributes to the total resistance $R(t)$ of the system. As it is the only component which is time dependent, it can be measured informing about the progress of the growth process. It can be

*This work is supported in part by the grant No. 223639203 of the Committee for Scientific Research. 
shown [3] that the time derivative of $R(t)$ is proportional to the growth rate $V_{\mathrm{gr}}$ if the dissolution of the back surface of the substrate by the contact melt can be neglected. Thus, the easy way to observe the changes of $V_{\mathrm{gr}}$ is obtained.

\section{Experimental results}

Thick AlGaAs epitaxial layers were grown by the modified LPEE method. All the details of the growth procedure and the graphite boat configuration can be found in $[1,2,4]$. The total resistance of the growth system measured at the growth temperature of $800^{\circ} \mathrm{C}$ was $\approx 68 \mathrm{~m} \Omega$. Only a fraction of this value $(\approx 6 \mathrm{~m} \Omega$ - as measured by the separate pair of voltage probes mounted to the boat) was the resistance of the growth cell. This clearly indicates that molybdenum rods, supplying the dc current to the cell, mainly contribute to the total resistance of our system. An accuracy of the measurements of the total resistance of the system was estimated to be $\pm 0.1 \mathrm{~m} \Omega$. This corresponds to the measurable thickness of the layer of $7 \mu \mathrm{m}$ or $29 \mu \mathrm{m}$ for undoped GaAs $\left(n=2 \times 10^{16} \mathrm{~cm}^{-3}, \rho_{800}=0.09 \Omega \mathrm{cm}[3]\right)$ or heavily doped GaAs:Si $\left(n=1.5 \times 10^{18} \mathrm{~cm}^{-3}, \rho=0.022 \Omega \mathrm{cm}\right.$ [3]), respectively. However, if only the resistance of the cell was used for in situ monitoring, the accuracy of the resistance measurements improved to leading to $\pm 0.01 \mathrm{~m} \Omega$-fold decrease in the measurable thickness of the layer.
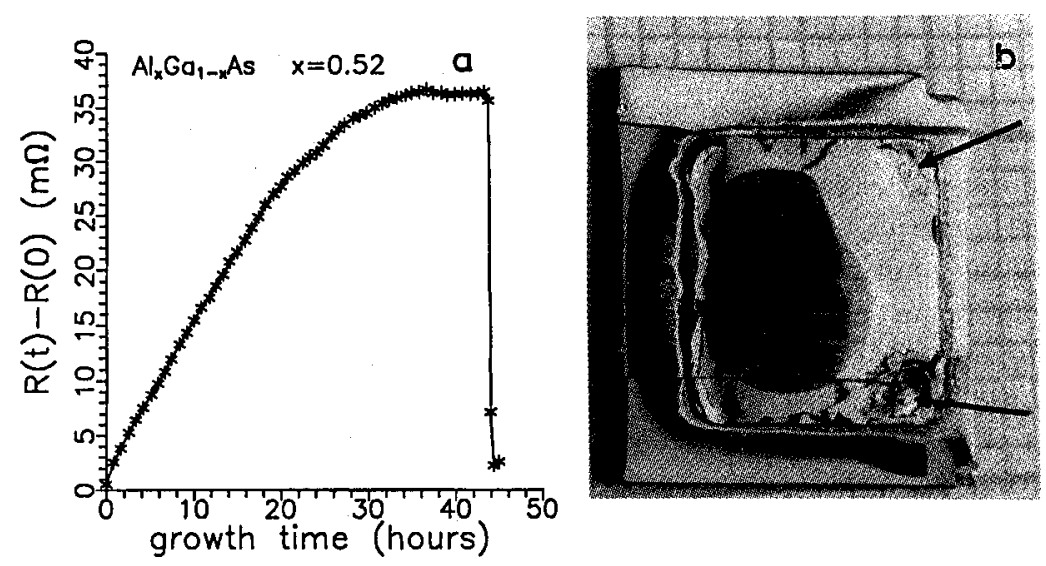

Fig. 1. (a) The growth cell resistance vs. growth time as measured during LPEE growth of thick AlGaAs epilayer whose picture is shown in (b). The holes etched through the epilayer as a result of system instability are marked with the arrows.

Figure 1a shows the time evolution of the growth cell resistance observed during the LPEE growth of $180 \mu \mathrm{m}$ thick $\mathrm{Ga}_{0.48} \mathrm{Al}_{0.52}$ As layer on GaAs substrate. As mentioned before, the slope $\mathrm{d} R / \mathrm{d} t$ can be used as a direct measure of the growth rate $V_{\mathrm{gr}}$. Thus, the initial continuous growth of the layer with the constant growth rate can be deduced from Fig. 1a. At this stage of the process the growth is fully controllable and $V_{\mathrm{gr}}$ is proportional to the electric current density. However, after approximately 25 hours of the growth $V_{\mathrm{gr}}$ decreases progressively and the 
epitaxial deposition stops as $R(t)$ saturates. The growth can be continued only if the electric current density $j$ forcing the crystallization is decreased. Otherwise, a growth instability occurs leading to local etching of the grown layer. This is clearly visible in Fig. 1b, where the as-grown surface of the layer obtained, is presented.

\section{Discussion}

In order to discuss the mechanism of the growth system instability presented above, let us consider the epilayer-substrate structure, in which local fluctuations of the epilayer thickness are present (Fig. 2). As a result some parts of the structure
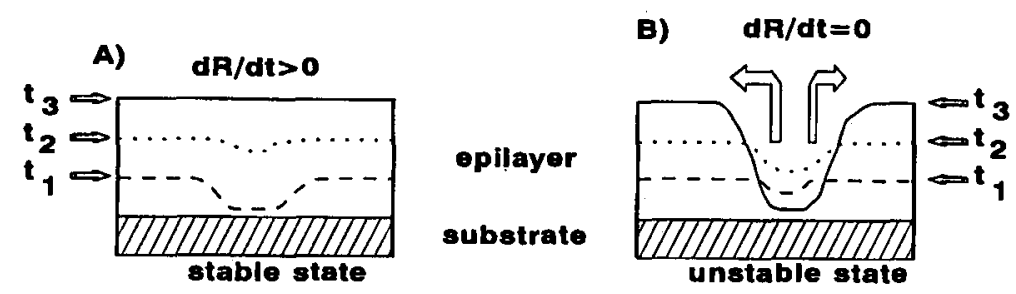

Fig. 2. The schematic drawing of the temporal evolution of the local fluctuation of epilayer thickness in stable (A) and unstable (B) state of the system.

are less resistant. Therefore, higher electric current density can be there expected. At the early stage of the crystallization procedure $V_{\mathrm{gr}}$ is proportional to $j$. The local increase in $j$ implies the local increase in $V_{\mathrm{gr}}$. Consequently, the fluctuations of the layer thickness decrease in time (stable growth). as shown in Fig. 2a. A quite different, unstable situation can occur in the $\mathrm{d} R / \mathrm{d} t=0$ state. Then, the local increase in $j$ is not accompanied with the local increase in As stream towards the substrate. Instead of that, local increase in the Joule heating is expected leading to etching through the structure and the short-circuit of the system as presented in Fig. 2b.

The discussed effect of system instability seriously limits the maximum thicknesses of AlGaAs epilayers obtainable by LPEE. It is well known that the growth kinetics of III-V epitaxial layers is limited by the velocity of $\mathrm{V}$ group element transport towards the substrate. Therefore, the growth self-termination observed during LPEE of AlGaAs can be understood as being caused by the existence of a barrier preventing As transport through the melt. Then the directed towards the substrate stream of As in the solution induced by the electric field is compensated by the opposite directed diffusive flux of As. There are evidences that the $\mathrm{Al}_{2} \mathrm{O}_{3}$ film formed on the surface of solutions containing $\mathrm{Al}$ may act as such a barrier for As transport.

The discussed instability of the growth system may be present in every growth configuration in which the condition $V_{\mathrm{gr}}=0$ is achieved by the compensation of two opposite directed streams of solute. For example, when the LPEE 
growth is performed with no feed material, the growth proceeds up to a certain thickness and then stops due to a gradual depletion of the solute in the solution. The maximum thickness of the epitaxial layer obtained $Z_{\max }$ is then used $[5,6]$ to measure fundamental parameters of the growth process (e.g. the effective mobility of solute, etc.). However, in the light of this study, care should be taken during such experiments because the system instability and the processes occurring in the $V_{\mathrm{gr}}=0$ state can seriously affect the value of $Z_{\max }$ and thus, the values of parameters studied.

\section{Conclusions}

In situ monitoring was found to be an effective and very useful tool to study the processes occurring during the LPEE growth of thick AlGaAs epitaxial layers. With the use of this technique the growth system instability has been detected and qualitatively explained.

\section{References}

[1] Z.R. Żytkiewicz, S. Miotkowska, J. Cryst. Growth 121, 457 (1992).

[2] Z.R. Żytkiewicz, S. Miotkowska, Acta Phys. Pol. A 82, 765 (1992).

[3] A. Okamoto, S. Isozumi, J. Lagowski, H.C. Gatos, J. Electrochem. Soc. 129, 2095 (1982).

[4] Z.R. Żytkiewicz, J. Cryst. Growth 131, 426 (1993).

[5] L. Jastrzębski, J. Lagowski, H.C. Gatos, A.F. Witt, J. Appl. Phys. 49, 5909 (1978).

[6] T. Bryskiewicz, J. Crystal Growth 43, 567 (1978). 